\title{
La inversión en investigación científica como medida del capital intelectual en las instituciones de educación superior
}

\author{
Maria I. Rojas ${ }^{(1) \star}$ y Ruby L. Espejo(2) \\ (1) Escuela de Economía, Universidad Pedagógica y Tecnológica de Colombia (UPTC), Tunja, Boyacá. \\ Colombia. (e-mail: maria.rojas03@uptc.edu.co) \\ (2) Escuela de Ciencias Humanísticas y Educación (UPTC) Tunja-Boyacá, Colombia (e-mail: ruby.espejo@uptc.edu.co)
}

${ }^{*}$ Autor a quien debe ser dirigida la correspondencia

Recibido May. 16, 2019; Aceptado Jul. 15, 2019; Versión final Ago. 20, 2019, Publicado Feb. 2020

\begin{abstract}
Resumen
Este estudio identifica una técnica para medir el capital intelectual en una institución de educación superior, basada en la evaluación de eficiencia de la inversión en investigación científica, a partir de los recursos económicos asignados, en relación con los componentes del capital intelectual: capitales humano, estructural y relacional. En un modelo de regresión ANCOVA con datos en panel, se relacionan los ingresos y egresos registrados a nivel contable. Se evidencia diferencia estadística significativa entre los tres componentes, siendo el capital estructural el más relevante. Esto permite medir la eficiencia en la gestión de los recursos, administrar e incentivar la competencia institucional en la gestión y transferencia de nuevo conocimiento a la sociedad.
\end{abstract}

Palabras clave: capital intelectual; investigación; gestión del conocimiento; capital estructural; capital humano

\section{The investment in scientific research as a measure of intellectual capital in higher education institutions}

\begin{abstract}
This study identifies a technique to measure the intellectual capital in a higher education institution, based on the evaluation of the efficiency of the investment in scientific research, through the allocated economic resources, in relation to the components of the intellectual capital: human capital, structural capital and relational capital. Using an ANCOVA regression model with panel data, the registered incomes and expenses are accounted. Significant statistical differences between the three components is evidenced, being the structural capital the most relevant. This allows demonstrating the efficiency in the allocation of the resources, management and incentives institutional competence in the management and transfer of new knowledge to the society.
\end{abstract}

Keywords: intellectual capital; research; knowledge management; structural capital; human capital 


\section{INTRODUCCIÓN}

La importancia de la producción científica en la actual economía del conocimiento ha guiado la atención del mundo académico y empresarial hacia la inversión en investigación científica, como un agregado de valor estratégico para las organizaciones (Mercado-Salgado, 2016). Estas ya no dependen solamente de los factores 'tierra', 'capital' y 'trabajo', sino también del factor 'conocimiento', considerado como el cuarto componente de la producción (Cypher y Dietz, 2014), y que se refleja en el capital intelectual (Martín-deCastro, et al., 2011). Gestionar ese conocimiento implica su medición, la que se equipara, por tanto, a las medidas financieras, y se orienta al proceso de toma de decisiones (Wann-Yih et al., 2008; Yi-Chu e YenChun, 2010) logrando ventajas competitivas no solo en función de los recursos tangibles, sino también de intangibles como el conocimiento y capacidad para transferirlo.

Diversos investigadores consideran que el capital intelectual es un activo intangible de las organizaciones, que debe ser valorado en correspondencia con sus objetivos, filosofía, dirección y políticas. Desde sus raíces, ha sido muy controversial (Moon y Kym, 2006); sin embargo, puede decirse que el capital intelectual es todo aquel conocimiento que aporta beneficio, según su utilidad y época (Bueno et al., 2011). En la economía, se referencia como un proceso de creación de valor que todavía está en una etapa emergente de desarrollo (Dean y Kretschmer, 2007). Teóricamente, se fundamenta en los estudios de la teoría de los recursos y las capacidades y se enmarca en diferentes disciplinas, tales como la Economía, Contaduría, Sociología, Finanzas, entre otras. Los indicadores de capital intelectual se encuentran construidos a partir de la información estadística obtenida de fuentes secundarias, que proveen las organizaciones, y de fuentes primarias, obtenidas por observación (Bueno et al., 2011; Mercado-Salgado, 2016). Mientras que, en el campo de la contabilidad, el capital intelectual se explica mediante la diferencia entre el valor contable y el valor de mercado de las empresas, hay quienes argumentan que en el balance contable no es mensurable el capital intelectual, por ser altamente subjetivo y difícil de medir.

Desde el punto de vista financiero, algunos autores consideran que el valor de la empresa, no puede ser medido como activo o pasivo tangible (Moon y Kym, 2006). Sin embargo, se siguen creando modelos con elementos comunes para hacer su medición de forma objetiva, usando cuestionarios, con los que se obtienen constructos que miden, directa o indirectamente, variables latentes cuantificadas sobre escalas y magnitudes operacionales, que permiten su comparación y percepción en diferentes dimensiones, con indicadores para capital humano, capital estructural y capital relacional (Labra y Sánchez, 2013).

Cuando no se mide y no se informa sobre el capital intelectual, se causa perturbación a la organización, que se traduce en costos excesivos de capital, produciendo deficiencias en la asignación de recursos y corriendo el riesgo de su incorrecta valoración. En este sentido, y en particular, la Organización para la Cooperación y el Desarrollo Económico, recomienda a las universidades públicas y centros de investigación generar reportes de capital intelectual, para mejorar la medición de sus contribuciones a la sociedad. Estos reportes, junto al panorama financiero y de gestión, complementan la información sobre la misión de las instituciones de educación superior, relativa a la generación y difusión de conocimiento (Bezhani, 2010; Sánchez et al., 2009). La información sobre capital intelectual permite optimizar los recursos disponibles para responder, desde la oferta formativa, a las demandas del contexto, a sus procesos de posicionamiento y acreditación, y a la justificación de inversión en investigación.

Dada la forma tripartita que se presenta el capital intelectual (Bueno et al., 2011; Labra y Sánchez, 2013), en las organizaciones, éste se apoya en el desarrollo del conocimiento y los recursos económicos para el desempeño organizacional que produce la imagen de valor agregado (Diez et al., 2010; Martínez, 2014). De igual manera, las instituciones educativas apalancan recursos y capacidades del talento humano, para sostenerse en condiciones favorables, asignando estratégicamente sus recursos económicos, financieros y sociales, gestionados de forma intencional. Además, a partir de la experiencia, las tecnologías y los procedimientos de enseñanza/aprendizaje posicionan su marca, reputación, cultura y capacidad competitiva dado que su naturaleza permite medir, describir y comparar las diferentes situaciones de forma estructurada y ordenada. Dicho posicionamiento asociado a la gestión y medición del capital intelectual parece provenir de la noción -intuitiva - de que el saber es algo más profundo que los simples datos o la información. Luego la gestión del conocimiento se piensa como aquella que contribuye con aspectos fundamentales para adaptarse y competir frente a todo tipo de cambios, sobretodo, a través de procesos organizacionales donde se persigue la combinación y la sinergia de las capacidades tecnológicas complementándose con el procesamiento de los datos y la información creativa e innovadora (Colunga et al., 2018).

En las Instituciones de Educación Superior (IES), el capital intelectual lo constituyen los procesos de investigación científica, la capacidad de innovación, los talentos, destrezas y el conocimiento tácito de sus miembros, como también el reconocimiento de la sociedad y colaboradores, todo lo cual sirve para transformar sus recursos materiales, financieros y humanos, en un sistema capaz de crear valor para los 
stakeholders (Sánchez et al., 2009), al combinar los tres componentes del capital intelectual: el humano, estructural y relacional. Por tanto, la visualización del capital intelectual permite a las IES rendir cuentas sobre los recursos que reciben de la sociedad. Esto significa, entonces, que la asignación estratégica de los recursos económicos y su producción en la investigación científica se hace medible al valorar la eficiencia en la distribución de los mismos (González y Rodríguez, 2010). En este contexto, el análisis y la divulgación logra transparencia, satisfacción en los usuarios y mejora la credibilidad, imagen y reputación de cualquier institución educativa.

Medir el capital intelectual permite a las organizaciones ver cómo la entrada de recursos, su transformación y salida, se refleja en el impacto de sus productos o servicios (Cortez, 2007). Coincide en esta apreciación Ríos y Castillo (2015) al evaluar la productividad y utilidad de la investigación sobre el impacto del conocimiento en lo social y económico. De acuerdo con Secundo et al. (2010), la investigación científica se relaciona con el capital intelectual cuando hay explotación del conocimiento e innovación, originando nuevas tecnologías que sirven de plataforma para aumentar la productividad, la competitividad y el crecimiento económico de una región. Para las organizaciones, incluyendo las IES, el éxito depende de la capacidad de adaptabilidad en relación con ritmos rápidos, que permiten asimilación estratégica moderna y que se asienten actitudes relativas a valores, creencias, cultura organizacional, y formación de conocimientos especializados aplicados a la innovación (Zlatea y Enache, 2015). Sin embargo, el principal motivo por el que algunas organizaciones, en particular, las de educación superior no miden, ni evalúan el capital intelectual es, quizá, por la falta de metodologías o modelos de gestión disponibles que identifiquen unificadamente estos activos.

Un modelo para valorar capital intelectual en universidades lo presenta Lagrosen et al. (2004), incluyendo indicadores de eficacia como número de administradores por cada profesor-investigador, número de investigaciones generadas por cada académico y la inversión en la capacitación del personal. El modelo de Alvarez-Aros y Bernal-Torres (2017) y el de Sánchez et al. (2009) reproducen metodologías aplicadas a empresas y centros de investigación, con fines de generar reportes para las universidades, que incluyan un seguimiento, a partir de los indicadores claves que incorporan el potencial humano y estructural. En estos modelos se vinculan la universidad, la empresa y otros organismos (Marulanda et al, 2016), en la gestión y difusión del conocimiento, lo que correspondería a un capital relacional.

Otras investigaciones que concuerdan al evaluar la gestión del capital intelectual en instituciones de educación superior con la buena distribución de los recursos de las actividades de investigación, se realiza a través de otros indicadores, como los de ciencia, tecnología e innovación -teniendo en cuenta el Manual de Frascati, aceptado por la mayor parte de países- y de los observatorios de ciencia y tecnología que cada institución posea, para ser comparados en cualquier situación (Rojas y Espejo, 2018). Estos, junto a los modelos Intelect e Intellectus de Euroforum, son ejemplos donde se explica el capital intelectual como riqueza de los intangibles en las organizaciones y en las instituciones educativas, examinando los tres componentes: capital humano, capital estructural -subdividido en los capitales organizativo y tecnológico- y el capital relacional -subdivido en capital de negocio y social-, que sirven de base para este estudio, pero midiendo, para el caso particular, los ingresos y egresos en los que incurren las IES al realizar actividades de investigación científica. En particular, la Universidad Pedagógica y Tecnológica de Colombia tiene en cuenta los valores éticos, la cultura y las bondades de la ciencia y la técnica como pilares de proyección social y económica, principalmente, en la formación de profesionales, investigación y la transferencia del conocimiento, buscando consolidar las capacidades en la producción de conocimiento científico, tecnológico y humanístico, que pueden ser medibles dentro de un modelo de capital intelectual.

Observando los tres capitales, desde el punto de vista de los administradores: el capital humano, se interpreta como el conocimiento tanto tácito como explícito, recogido principalmente sobre los saberes y capacidades de los miembros de las comunidades que lo dominan, conceptuado en competencias, talento y experiencia aplicada (Gujarati y Porter, 2010).. Así en la empresa, el efecto de la experiencia laboral y los años de educación formal se retornan en el ingreso (salario). Esto implica que la educación en el mercado se traduce en mayores salarios. En este sentido, los costos adicionales en la formación influyen a la hora de seguir los estudios, lo cual amplía las diferencias compensatorias de los ingresos, por lo que el salario pagado a las personas que desarrollan actividades de investigación científica, directa o indirectamente, en las IES, se cuantifica para medir el capital humano (Martínez, 2014). Además, Los activos centrados en el individuo y las cualificaciones que lo conforman (Espejo, 2015) no pueden ser propiedad de la organización, sino que se convierten en motor y energía, fuente de ventaja competitiva sustentable; y su inversión según Becker (1994) define una actividad que repercute sobre la renta monetaria, pudiendo ser adquirida por la escolarización, formación en el empleo, atención médica y adquisición de información sobre el sistema económico. 
El capital estructural representa el capital organizativo de naturaleza explícita e implícita; sus elementos constitutivos son la cultura, el aprendizaje, el desarrollo de actividades organizacionales y la actividad productiva tangible e intangible. En el contexto universitario se identifica por la infraestructura básica y por los recursos generadores de datos empíricos primarios, esto es, todos y cada uno de los recursos puestos de manifiesto en bibliografías, compilaciones documentales, bases de datos, sistemas de gestión, desarrollo de nuevas tecnologías, patentes y material de laboratorios; es decir, recursos para transformar procesos del conocimiento, ya sea de forma tácita o explícita (Bueno et al., 2011; Hernández et al., 2015; González y Rodríguez, 2010). El capital tecnológico también forma parte de lo estructural, y es el conocimiento aplicado que la organización o institución logra explicitar, sistematizar y socializar; es el soporte logístico, donde se refleja la eficiencia y eficacia de la organización; es la tecnología organizativa propia de los empleados en su trabajo (Bueno et al., 2011). En el contexto universitario (Bueno et al., 2011) se relaciona con el esfuerzo en investigación y desarrollo, dotación tecnológica y propiedad intelectual e industrial.

El capital relacional está representado en el capital de negocio, la vinculación entre la organización y sus stakeholders y el capital social, con lo que expresa el nivel de integración, compromiso, cooperación, cohesión, conexión y responsabilidad social. Permite a la organización desarrollar actividades con el Estado y los restantes agentes sociales que actúan en su entorno, para generar valor de marca y nuevos investigadores. En el contexto universitario, se incorporan los rubros que, cuantitativa y cualitativamente, se relacionen económicamente con políticas institucionales que mantienen la entidad con los diferentes agentes y su entorno socioeconómico. Sus elementos incluyen la inversión en: comunicación, relación comercial entre clientes y proveedores, marketing en las website y extranets, colaboración con las empresas y otras instituciones públicas con las que realiza proyectos de investigación. Otros componentes de gran relevancia son la pertenencia a sociedades científicas y la participación en reuniones científicas, las cuales se evidencian en la vinculación a redes, en membrecías en comunidades científicas y en la participación de los grupos de investigación en eventos científicos e investigativos nacionales e internacionales (Casanueva y Gallego, 2010; Bueno et al., 2011).

\section{METODOLOGÍA}

Abordando las teorías organizacionales en las que se sustenta la noción de capital intelectual y algunas técnicas econométricas, es posible responder a la pregunta sobre cómo se dinamiza la gestión de inversión en capital humano, estructural y relacional, en las instituciones de educación superior, con relación a la investigación científica. El estudio es de tipo descriptivo y exploratorio - dado que el interés se centra en explicar por qué dos o más variables están vinculadas- mediante un modelo de regresión Ancova con datos en panel, dirigido a responder las causas del evento descrito, determinando las características del objeto por investigar y sus diferencias. La muestra es fija, puesto que los datos están conformados por 39 registros u observaciones que, por su naturaleza, son recopilados desde enero de 2003 a diciembre de 2015, y corresponden a los egresos e ingresos registrados en la contabilidad de los rubros ejecutados específicamente en investigación científica de la UPTC, suministrados por la oficina de presupuesto.

El procedimiento consiste en tres etapas: Primero, se mide la gestión del capital intelectual mediante un indicador de eficiencia, puesto que es un constructo subyacente que no se puede medir de forma directa, sino mediante manifestaciones externas; por lo anterior se utiliza la combinación de variables medibles: los egresos que se han ejecutado en un periodo, dividido por los ingresos asignados a la investigación científica, expresada por la relación Egresos/Ingresos = Eficiencia. Segundo, se clasifican los rubros de inversión en investigación científica de la universidad en variables proxy del capital intelectual, identificados en cada uno de los tres componentes, que muestra la Tabla 1: capital humano, capital estructural y capital relacional. Tercero, se estima el modelo de regresión ANCOVA, para lo cual se define la variable dependiente como la eficiencia real en inversión del capital intelectual, obtenida mediante el logaritmo natural de la eficiencia en unidades constantes $\operatorname{Ln}($ Eficiencia) $=\mathrm{Ln}($ Egresos/Ingresos) (los valores de los ingresos y los egresos se transforman a precios constantes deflactados por el IPC mensual, año base: 2008, consolidado por el Banco de la República de Colombia); y como variables independientes, la inversión en: capital humano, capital estructural y capital relacional, y la covariable tiempo denominada tendencia (años 2003 a 2015). Teniendo en cuenta que los efectos de la inversión en investigación son a largo plazo, se hace necesario incluir la variable tiempo. Además, el conocimiento llega al consumidor en forma lineal; de acuerdo con las teorías de Bueno et al. (2011) y Martínez (2014), las categorías (componentes) del capital intelectual reflejan una dinámica acumulativa, lo cual establece que el modelo sea lineal aditivo. La función del modelo se define mediante la ecuación de un modelo log-lin derivado de una función de producción tipo Coob-Douglas, representada por la ecuación (1):

\section{Eficiencia $=F($ capital intelectual, tiempo $)$}

$(\text { LnEficiencia })_{i t}=\alpha_{1 i}+\alpha_{2} D_{2 i}+\alpha_{3} D_{3 i}+\beta_{1} T_{i t}+U_{i t} \quad i=1,2,3, \ldots, 11$ 
Donde las variables dicótomas $D_{2 i}=1$, si la observación corresponde al capital humano $C . H$, y 0 en otro caso; $D_{3 i}=1$, si la observación corresponde al capital relacional $C . R$, y 0 en otro caso.

Tabla 1. Variables indicadoras de capital intelectual clasificado por los recursos asignados a la investigación científica

\begin{tabular}{|c|c|c|}
\hline Capital humano & $\begin{array}{l}\text { Capital estructural: Organizativo y } \\
\text { Tecnológico }\end{array}$ & Capital relacional: De Negocio y Social \\
\hline $\begin{array}{l}\text { Salarios del personal } \\
\text { vinculados a la } \\
\text { Dirección de } \\
\text { Investigación: } \\
\text { Horas extras, subsidio } \\
\text { de transporte. } \\
\text { Prestaciones sociales: } \\
\text { primas, cesantías, } \\
\text { intereses a de las } \\
\text { cesantías, vacaciones. } \\
\text { Honorarios del personal } \\
\text { vinculado a la Dirección } \\
\text { de Investigación. } \\
\text { Viáticos del personal } \\
\text { vinculado a la Dirección } \\
\text { de Investigación. } \\
\\
\text { Seguridad social y } \\
\text { parafiscal del personal } \\
\text { vinculado a la Dirección } \\
\text { de Investigación. }\end{array}$ & $\begin{array}{l}\text { Formación de docentes, jóvenes } \\
\text { investigadores y semilleros. } \\
\text { Biblioteca: bases de datos, libros, } \\
\text { compilaciones, documentales. } \\
\text { Sistemas de gestión: servicios } \\
\text { administrativos. } \\
\text { Desarrollo de nuevas tecnologías. } \\
\text { Patentes. Adquisición de elementos en las } \\
\text { facultades, institutos y laboratorios. } \\
\text { Infraestructura básica: edificios, } \\
\text { instalaciones, para la investigación } \\
\text { laboratorios y otras dependencias. } \\
\text { Entornos de aprendizaje, capacitación y } \\
\text { transmisión de conocimientos: } \\
\text { Congresos, simposios, seminarios y otros. } \\
\text { Fomento a la innovación, investigación y } \\
\text { desarrollo, ingeniería, estudio de mercados } \\
\text { y de usuarios, planificación financiera, } \\
\text { diseño de productos y procesos, producción } \\
\text { industrial, canales de distribución, } \\
\text { proveedores de equipos y materias primas, } \\
\text { ventas. } \\
\text { Gastos de los centros de investigación y } \\
\text { universidad para impulsar la investigación. }\end{array}$ & $\begin{array}{l}\text { Proyectos entidades estatales y privadas: } \\
\text { alcaldías y Gobernación de Boyacá, } \\
\text { Corpoboyacá, Ecopetrol y otros. } \\
\text { Actividades de comunicación y relaciones } \\
\text { públicas. Actividades de marketing, } \\
\text { Website, extranet, vinculación de redes. } \\
\text { Membrecía en comunidades científicas. } \\
\text { Participación en eventos científicos } \\
\text { nacionales e internacionales. } \\
\text { Defensa del medioambiente. } \\
\text { Procesos de elaboración de normas. } \\
\text { Comisiones, participación en la } \\
\text { formulación de programas a largo plazo. } \\
\text { Canales de difusión para promocionar los } \\
\text { resultados. } \\
\text { Relaciones con aliados: acuerdos de } \\
\text { colaboración de investigadores o grupos } \\
\text { de investigación con otros equipos de } \\
\text { investigación. } \\
\text { Vigilancia competitiva y procesos de } \\
\text { relación con competidores. } \\
\text { Procedimientos tecnológicos que facilitan } \\
\text { el desarrollo, en tiempo y forma, de las } \\
\text { relaciones con el entorno. }\end{array}$ \\
\hline
\end{tabular}

Como se trata de tres tipos de capital, solo utilizamos dos variables dicótomas, dejando el capital estructural C.E, como categoría base o de referencia; $\alpha_{1 i}=\alpha_{1 i}+U_{i t}$ es el intercepto; $\alpha_{-i}$ indica el valor de la mediana común del intercepto de cada capital, y $U_{i t}$ refleja los diferentes efectos individuales en el intercepto de cada tipo de capital. Los demás coeficientes $\alpha$ representan el grado en que los valores del intercepto de los demás tipos de capital difieren del valor del intercepto del capital estructural. Los coeficientes son $\alpha_{j}$ y $\beta_{j}$ son parámetros e indican los efectos de las variables con respecto al índice de eficiencia (real), y $T_{i t}$ indica el tiempo (año) en el que se ejecutan los rubros de los egresos e ingresos del capital intelectual. $\beta_{1}$ representa la tendencia en porcentaje del crecimiento/decrecimiento en la eficiencia de la inversión en capital intelectual a través del tiempo (Gujarati y Porter, 2010). Antes de realizar el análisis Ancova, se hace un análisis de varianza ANOVA y se complementa con la estimación de un modelo de regresión lineal de covarianza en panel balanceado.

En las hipótesis del modelo, en una organización, la eficiencia en el uso del capital intelectual interactúa de forma circular y recíproca positiva. El capital humano tiene un efecto positivo sobre el capital estructural, en el sentido en el que el capital humano es la fuente de innovación y renovación estratégica, construyendo al capital estructural, que puede verse como consecuencia de la creatividad humana; el capital estructural es el conocimiento incrustado en las rutinas de la organización (Martínez, 2014). Al explorar la relación entre el capital relacional y el estructural en una empresa, el capital estructural tiene un efecto positivo sobre el capital relacional; los beneficios a largo plazo se crean por la fusión de los dos capitales, identificados como intangibles. El capital relacional se define aquí como el conocimiento incrustado en la cadena de valor de la organización, es decir, el conocimiento identificado en la relación de la organización con sus proveedores, clientes y entidades fuera de la organización (Martínez, 2014).

El capital relacional tiene un efecto positivo sobre el capital humano. La capacidad de una empresa para interactuar positivamente con miembros de la comunidad empresarial estimula el potencial de sus miembros y así crea riqueza, es decir, aumenta el capital humano y el estructural. El ambiente de tales organizaciones 
cambia de la misma manera que cambia la relación con sus clientes; este cambio obliga a las personas a desarrollar nuevas habilidades y les permite adaptarse a nuevas situaciones (Martínez, 2014). De acuerdo con el objetivo y las hipótesis a comprobar, se realiza un análisis de varianza ANOVA, para encontrar diferencia estadística significativa entre los tres componentes del capital intelectual y diferenciar, "dos a dos", su comportamiento. Luego, para evidenciar si el capital estructural es el más relevante entre los componentes se realiza un análisis mediante la regresión ANCOVA. Los datos se procesan con el paquete econométrico Eviews V.9 e I.B.M - S.P.S.S V.20.

\section{RESULTADOS}

El análisis estadístico descriptivo del comportamiento de las variables en estudio sobre los componentes del capital intelectual, en términos de eficiencia, se resume en la figura 1 -Tendencia del crecimiento en eficiencia capital intelectual, años 2003 a 2015-, observando cuatro cambios, entre el 2003 y el 2015, con tendencia dispersa. Desde el 2003 hasta finales del 2008, el capital humano presenta alto índice de eficiencia sobre el capital estructural y relacional. El mismo comportamiento se evidencia desde el 2012 a 2014. Mientras que desde el 2009 al 2012, y desde el 2014 a 2015, último periodo en estudio, es la eficiencia del capital estructural la que sobresale, recibiendo el efecto del capital humano y relacional de los 5 años anteriores.

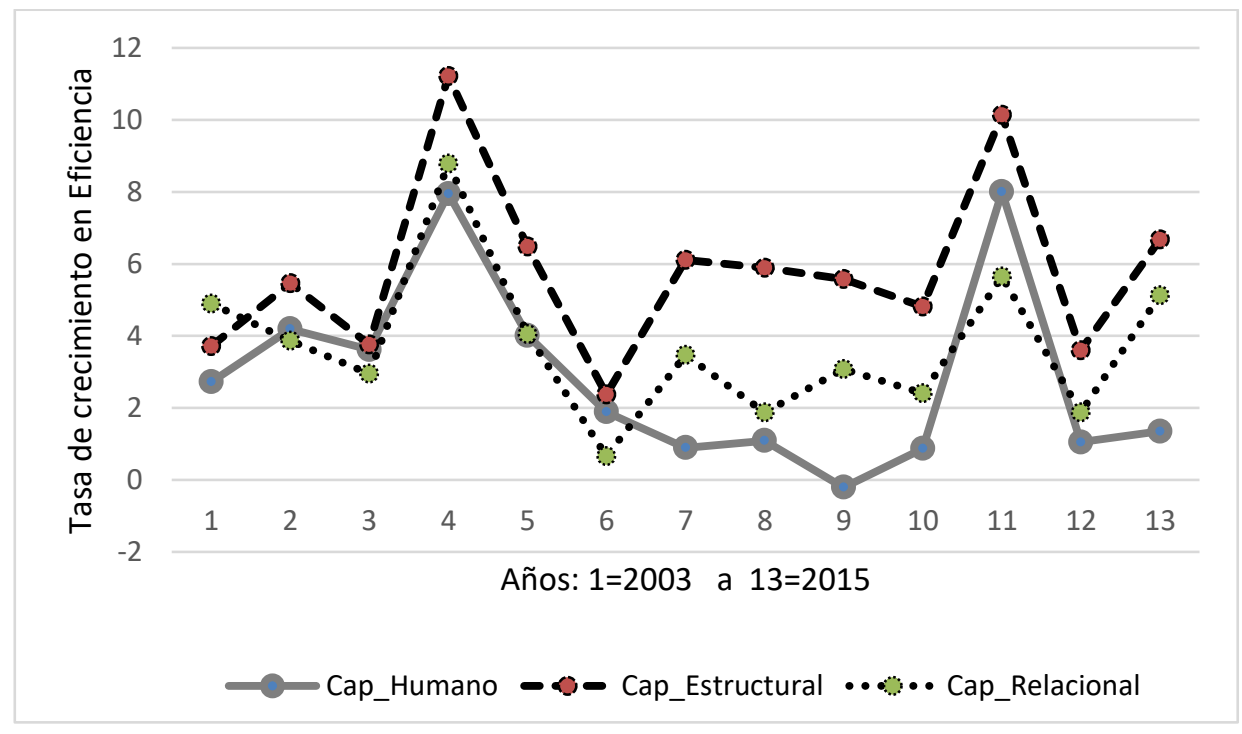

Fig. 1: Tendencia del crecimiento en eficiencia capital intelectual, años 2003 a 2015

Para comprobar si existe diferencia significativa entre las tres categorías de la variable Capital intelectual se realiza el análisis de varianza ANOVA (Tabla 2). Antes, se comprueba el cumplimiento de los supuestos de igualdad de varianzas en los grupos, y de normalidad en los datos del capital intelectual. La prueba de homogeneidad de la varianza, el valor $p$ de significancia para el estadístico de Levene, con 0,071 , y para la prueba de normalidad de Shapiro-Wilk y Kolmogorov-Smirnov, con valores cercanos a 0,4 , que son mayores a 0,05 (nivel de significancia con el que se compara), indican el cumplimiento de homoscedasticidad, para el primer caso, y, para el segundo, que los datos provienen de poblaciones normales.

Ahora, el estadístico $F$ es calculado por el cociente entre los dos estimadores de la varianza poblacional, los cuales se obtienen a partir de la variación existente entre las medias de los grupos (variación intergrupos) y la variación existente entre las puntuaciones dentro de cada grupo (variación intragrupos). Si el estadístico $F$, junto con su nivel observado o valor de significancia, es igual a cero (0), menor que 0,05 , es indicativo de que se debe rechazar la hipótesis de igualdad de medias, muestra de ello -Análisis de varianza para los componentes del capital intelectual: variable LnEficiencia- la tabla 2.

Tabla 2: Análisis de varianza para los componentes del capital intelectual: variable LnEficiencia

\begin{tabular}{|l|c|c|c|c|c|}
\hline Fuente de variación & Suma de cuadrados & Grados de libertad & Media cuadrática & $F$ & Sig. \\
\hline Intergrupos & 216,85 & 2 & 108,42 & 25,75 & 0,00 \\
\hline Intragrupos & 151,55 & 36 & 4,21 & & \\
\hline Total & 368,40 & 38 & & & \\
\hline
\end{tabular}


Por tanto, se puede concluir que las poblaciones definidas por las variables correspondientes a los componentes del capital intelectual no tienen el mismo promedio de inversión; hay al menos una población cuyo promedio en inversión difiere de otro, lo cual se relaciona con la figura 1. Tendencia del crecimiento en eficiencia capital intelectual, años 2003 a 2015-. Dado que existen diferencias estadísticamente significativas entre los componentes del capital intelectual, la prueba HDS de Tukey permite comparar, "dos a dos", la diferencia entre las categorías de los componentes del capital intelectual, como se muestra en la Tabla 3: capital humano (1) y capital estructural (2); capital humano (1) y capital relacional (3); capital relacional (3) y capital estructural (2).

Tabla 3. Prueba de Tukey. Comparaciones Múltiples: variable dependiente LnEficiencia

\begin{tabular}{|c|c|c|c|c|c|c|}
\hline (I) capital & (J) capital & Diferencia de medias (I-J) & Error típico & Sig. & \multicolumn{2}{|c|}{ Intervalo de confianza al 95 \% } \\
\cline { 5 - 7 } & & & & & Límite inferior & Límite superior \\
\hline \multirow{2}{*}{ 1 Humano } & Estructural (2) & $-0,07$ & 0,80 & 0,99 & $-2,04$ & 1,89 \\
\cline { 2 - 7 } & Relacional (3) & $0,96^{*}$ & 0,80 & 0,00 & 2,99 & 6,93 \\
\hline \multirow{2}{*}{2 Estructural } & Humano (1) & 0,07 & 0,80 & 0,99 & $-1,89$ & 2,04 \\
\cline { 2 - 7 } & Relacional (3) & $5,03^{*}$ & 0,80 & 0,00 & 3,07 & 7,00 \\
\hline \multirow{2}{*}{ 3 Relacional } & Humano (1) & $-4,96^{*}$ & 0,80 & 0,00 & $-6,93$ & $-2,99$ \\
\cline { 2 - 7 } & Estructural (2) & $-5,03^{*}$ & 0,80 & 0,00 & $-7,00$ & $-3,07$ \\
\hline
\end{tabular}

Evidentemente, con la prueba de Tukey puede comprobarse que existe diferencia significativa estadísticamente entre la eficiencia en inversión del capital humano (1) y la del capital relacional (3), superando el primero al segundo en 4,9 puntos; así como entre la del capital estructural (2) y la del capital relacional (3), superando el primero al segundo en 5,03; lo que no ocurre con la eficiencia en inversión en el capital relacional (3), que se encuentra por debajo de la del capital humano y la del estructural con 4,96 y 5,03 puntos, respectivamente. (Los grupos cuyas medias difieren significativamente con el nivel de significación establecido —0,05 por defecto— están marcados con un asterisco).

El análisis gráfico y descriptivo antes mencionado se reafirma con el análisis econométrico, mediante la estimación del modelo de panel Log-Lin, ecuación (1), considerando inicialmente un panel de coeficientes constantes, con el fin de medir la sensibilidad de la eficiencia en función del capital y del tiempo. Mediante el método Mínimos cuadrados ordinarios y haciendo uso de una función potencial tipo Coob-Douglas calcula directamente las semielasticidades de los tipos de capital respecto de la eficiencia (Gujarati y Porter, 2010). Antes, se examina el cumplimiento de los supuestos del modelo, mediante valores $p$ (probabilidad mínima con la cual se rechaza la hipótesis nula de la prueba en consideración, para ser comparada a un nivel de significancia del $5 \%$ ). La prueba de normalidad para datos en panel, dada por el estadístico de Jarque Bera, y el $p$ (con valor $0,315>0,05$ ) no rechazan la hipótesis de normalidad de los errores; la prueba de homogeneidad, dada por el estadístico Levene para grupos $(p=0,07>0,05)$ no rechaza la hipótesis de homogeneidad de los errores; y la prueba de no autocorrelación o dependencia entre errores, cuyo valor $p$ de significancia del estadístico de Breusch-Pagan LM es $0,13>0,05$, no rechaza la hipótesis. Lo que indica el cumplimiento de las pruebas en los errores.

Por la prueba de raíz unitaria para los residuos de la regresión se observa que las variables índices de eficiencia en las categorías del Capital intelectual comparten una tendencia común, dada la probabilidad del estadístico Levin, Lin y Chu $t^{*}(0,0252)$ y chí-cuadrado de la prueba de Phillips Perron PP-Fisher $(0,0022)$. Comparado con un nivel de significancia del $5 \%$, indica que se rechaza la hipótesis nula de que exista raíz unitaria, es decir, las series están cointegradas. En términos económicos, significa que las variables están cointegradas, si existe una relación de largo plazo o de equilibrio entre ambas (Gujarati y Porter, 2010). El modelo estimado se ajusta según la ecuación 2 :

LnEficiencia $=3,60-0,49{ }^{*} \mathrm{C} . \mathrm{H}-4,61745361^{*} \mathrm{C} . \mathrm{R}-0,03^{\star}$ Tendencia

$$
P \text { - Valor }=(0,00) \quad(0,68) \quad(0,001) \quad(0,47)
$$

$R^{2}=0,59 \quad d=1,58$

En términos de semielasticidad, el modelo de la ecuación (2) se interpreta de manera directa mediante el proceso de Halvorsen y Palmquist (Gujarati y Porter, 2010). El capital humano presenta una semielasticidad de $-39,15 \%$, siendo esta variable no significativa, comparado el valor $p$ prob. 0,68 a un nivel de significancia del $5 \%$, ceteris paribus. Mientras que el capital relacional presenta alta semielasticidad en índice de eficiencia, indicando que la mediana es menor que la del capital estructural en un 99,01\%, siendo esta 
variable significativa estadísticamente a un nivel de significancia del $5 \%$ (comparado este valor con prob. 0.0001), manteniendo los demás factores constantes (Gujarati y Porter, 2010).

El intercepto o constante [ecuación (2)] representa la mediana del índice de eficiencia en inversión del capital relacional. En el periodo de estudio, se registró un índice de \$36,64 (pesos constantes); por debajo de este valor se alcanza el $50 \%$ de las metas propuestas para desarrollar capital intelectual. La variable tendencia - aun cuando no es significativa estadísticamente a un nivel del $5 \%$, comparado al valor prob. 0,4729 - respecto al índice de eficiencia de la inversión en capital intelectual, indica que, al pasar un año, este índice tiende a disminuir en un 3,2 \%, ceteris paribus. A priori, se esperaba que la relación entre el factor tiempo (años) y la eficiencia de inversión en investigación pudiera ser positiva o negativa, ya que depende de las políticas de inversión implementadas y del flujo de ingresos por año de los convenios realizados con anterioridad. Este capital, en la universidad, se hace invisible en el tiempo, dado que los beneficios de la ciencia son intangibles y se revelan indirectamente con el tiempo; por esto, tal vez, al momento no se hace significativo.

En una segunda etapa, se estima el modelo en panel con efectos fijos y aleatorios sin la variable tendencia, por no ser significativa. Observemos la estimación con efectos fijos dada en la ecuación 3 y tabla 4:

LnEficiencia $=2,95-0,07^{*} \mathrm{C} \cdot H-5,03925805921^{*} \mathrm{C} . R+[P E R=F]$

Valor $p=(0,00)(0,95) \quad(0,00)$

$R^{2}=0,63 d=1,47$

Con el fin de seleccionar el modelo que mejor se ajusta a los datos, se aplica el Test de Hausman, cuyo valor $p$ de probabilidad del estadístico chi-cuadrado igual a 1, que comparado a un nivel de significancia de $5 \%$ es mayor, indica que el modelo más adecuado se da con efectos aleatorios.

Tabla 4. Efectos Aleatorios PER=F

\begin{tabular}{|l|l|l|l|l|l|l|l|}
\hline Fecha & $01 / 01 / 2003$ & $01 / 01 / 2004$ & $01 / 01 / 2005$ & $01 / 01 / 2006$ & $01 / 01 / 2007$ & $01 / 01 / 2008$ & $01 / 01 / 2009$ \\
\hline Efecto fijo & 0,38 & 0,03 & $-0,26$ & 1,67 & 0,10 & $-1,03$ & $-0,09$ \\
\hline Fecha & $01 / 01 / 2010$ & $01 / 01 / 2011$ & $01 / 01 / 2012$ & $01 / 01 / 2013$ & $01 / 01 / 2014$ & $01 / 01 / 2015$ & \\
\hline Efecto fijo & $-0,62$ & $-0,22$ & $-0,44$ & 0,63 & $-0,62$ & 0,46 & \\
\hline
\end{tabular}

Luego, se estima el modelo con efectos aleatorios observados en la ecuación (4) y tabla 5, para la interpretación final.

LnEficiencia $=2,95-0,07^{*} \mathrm{C} . H-5,03^{*} \mathrm{C} . R+[P E R=R]$

Valor $p=(0,00)(0,94) \quad(0,00)$

$R^{2}=0,58 \quad d=1,57 P$ valor estadístico $F=0$

Tabla 5. Efectos $P E R=R$

\begin{tabular}{lccccccc}
\hline Fecha & $01 / 01 / 2003$ & $01 / 01 / 2004$ & $01 / 01 / 2005$ & $01 / 01 / 2006$ & $01 / 01 / 2007$ & $01 / 01 / 2008$ & $01 / 01 / 2009$ \\
\hline $\begin{array}{l}\text { Efecto } \\
\text { aleatorio }\end{array}$ & 0,0 & 0,0 & 0,0 & 0,0 & 0,0 & 0,0 & 0,0 \\
\hline Fecha & $01 / 01 / 2010$ & $01 / 01 / 2011$ & $01 / 01 / 2012$ & $01 / 01 / 2013$ & $01 / 01 / 2014$ & $01 / 01 / 2015$ \\
\hline $\begin{array}{l}\text { Efecto } \\
\text { aleatorio }\end{array}$ & 0,0 & 0,0 & 0,0 & 0,0 & 0,0 & 0,0 \\
\hline
\end{tabular}

Los resultados de la ecuación (4) indican significatividad individual en las variables explicativas (exógenas), si se tiene en cuenta que los valores $p$ asociados a la estadística de prueba $t$ son iguales a cero (0), menores a 0,05, nivel de significancia al cual se comparan; a excepción de la categoría Capital humano $(0,94$ variable $\mathrm{C} . \mathrm{H})$, no existe diferencia significativa frente a la categoría de referencia (Capital estructural variable C.R). En conjunto, los coeficientes estimados presentan en el modelo una buena estimación, observando el valor $p$, valor de significancia igual a cero del estadístico $F$.

De igual forma que con los modelos estimados anteriormente, se comprueba que cumple los supuestos de normalidad, homoscedasticidad, no multicolinealidad y exogeneidad. En términos de semielasticidad, se 
interpreta de manera directa mediante el proceso de Halvorsen y Palmquist (Gujarati y Porter, 2010). El capital humano presenta una semielasticidad de $-7,92 \%$, lo cual indica que la mediana de la eficiencia del capital humano es menor respecto al capital estructural en aproximadamente $7,92 \%$, ceteris paribus. El capital relacional presenta alta semielasticidad en índice de eficiencia, indicando que la mediana en inversión es menor que la del capital estructural en un 99,3\%, manteniendo los demás factores constantes. A priori, se obtienen los signos esperados, en relación con el índice de eficiencia en la inversión del capital intelectual, y sus componentes observan el impacto argumentado por Martínez (2014).

\section{DISCUSIÓN}

Desde la perspectiva económica de la inversión en investigación científica, se confirma la hipótesis planteada para este estudio: los recursos de una institución superior, especialmente los intangibles, representados y medidos en los componentes de capital intelectual, son como los de una empresa propensa (unos más que otros) a obtener y mantener un rendimiento superior o igual al invertido. De ahí que los resultados presenten diferencia significativa en los tres componentes: capital humano, estructural y relacional. El más relevante para una institución educativa es el capital estructural, componente clave para la innovación, investigación y desarrollo, que se manifiesta en lo que se sabe hacer, incluyendo lo personal, la cultura, lo organizacional y lo tecnológico, como se explica en las teorías de Sánchez et al. (2009); Labra y Sánchez (2013); Colunga et al. (2018). Además, los resultados de la prueba de raíz unitaria indican que existe una relación de equilibrio a largo plazo, que, en términos económicos, define la eficiencia que se le ha dado a la distribución de los recursos (Wann-Yih et al., 2008).

Cabe señalar que el capital intelectual permite lograr la eficiencia, pese a la fluctuación de la inversión en investigación científica. Teniendo en cuenta que las IES son organizaciones cuya meta es la producción y difusión del conocimiento, al comparar los resultados de la significatividad estadística superior del capital estructural respecto al humano y al relacional, se confirma que las instituciones educativas son altamente competitivas al aprovechar los recursos humanos, tecnológicos y financieros para ayudar a potenciar sus recursos en conjunto y la transferencia de los conocimientos a la sociedad. Así mismo, en el campo gerencial, según Carro-Suárez et al. (2017), esta consideración constituye un paso para reconocer el papel de la innovación y los efectos del apalancamiento con los que opera la institución.

No obstante, y de acuerdo con el trabajo de Martínez (2014), el capital humano es el inmediato precursor de la riqueza intelectual de la organización. Tanto el conocimiento de las personas que trabajan en la organización como el capital relacional utilizado resulta nuevamente en desarrollo de conocimientos, capacidades y habilidades, convirtiéndose en capital estructural, generando que el capital intelectual esté continuamente en desarrollo, si se tiene en cuenta que cada uno de sus componentes tiene una incidencia sobre el resto, de tal manera, que, un cambio en cualquiera de ellos produce un aumento/disminución en los demás componentes. La hipótesis sobre los componentes del capital intelectual también se examina bajo la semielasticidad.

La diferencia en inversión en capital humano y estructural no es estadísticamente significativa; esto indica que los dos interactúan conjuntamente para estimular el potencial en la creación de riqueza. Sin embargo, se destaca que el más relevante de los componentes para una institución educativa es el capital estructural, si se tiene en cuenta los signos negativos en el análisis econométrico de los coeficientes, antes mencionados, que indican que la eficiencia de la inversión en capital humano es inferior a la del capital estructural. De acuerdo con Min-Wei y Barry (2006), las instituciones son altamente competitivas, al aprovechar sus recursos humanos, tecnológicos, financieros y culturales para potenciarlos en las diferentes dimensiones, estrategia que ayuda a transferir los conocimientos a la sociedad y a incrementar los recursos para maximizar la productividad desde una perspectiva distinta al impacto que produce lo meramente académico como eje de sustentabilidad.

Cuando se presenta una inversión sostenida, con la ayuda de políticas públicas de estímulo a la investigación y al desarrollo, resulta oportuna la interacción de los tres capitales. La conexión entre la inversión en capital humano y estructural con el capital relacional, mediante la colaboración de proyectos de investigación entre instituciones, contratos suscritos con empresas y otros proyectos gestionados, hace competitivas a las IES. Los conocimientos son elemento clave en la dinamización del proceso de obtención, generación, aplicación y apropiación del nuevo conocimiento representado en el capital estructural. Por tanto, se debe favorecer la acumulación de capital humano, que genere un flujo de ingresos en el tiempo. Una estrategia para dicho favorecimiento lo constituyen las políticas encaminadas a aumentar el intercambio de conocimientos. Según Zlatea y Enacheb (2015) la interdependencia entre el capital humano y el desempeño organizacional en las universidades sugieren que cada vez se esté más interesado en atraer y mantener el personal apropiado, que sea capaz de crear e innovar, previendo la compatibilidad con su cultura y la formación de conocimientos especializados. 


\section{CONCLUSIONES}

El análisis más significativo en los procesos de los componentes del capital intelectual se refleja con mayor visibilidad en el capital estructural, con un nivel superior respecto a la eficiencia de la inversión en capital relacional y humano. Al combinar la inversión de los componentes del capital intelectual, éstos están unos más que otros propensos a revertir su inversión o a mantener su rendimiento superior al invertido, dependiendo de los procesos de gestión, que se le haya dado. A partir de éste resultado, permite a las IES, identificar, medir y administrar uno de sus activos intangibles, como es la investigación científica que desarrolla el personal encargado de producirla. Además, se puede plantear un concepto de gestión en capital intelectual en función de transformar el nuevo conocimiento con valor agregado.

Desde el punto de vista estratégico, el capital intelectual es un factor crucial de beneficio a largo plazo; su aplicación es ampliamente aceptada, en las IES, como capacidad para innovar. Los retornos del capital estructural dan cuenta de que el rendimiento está estrechamente relacionado con la capacidad para utilizar, de forma efectiva, los recursos: el desarrollo del capital humano y la consecución del capital relacional ameritan una importante gestión por parte de las IES.

Mediante el modelo econométrico de Regresión Ancova con datos en panel, recopilando datos de los ingresos y egresos contables, y a través de un análisis de varianza, se ofrece soporte empírico para evaluar la gestión de inversión en capital intelectual en instituciones de educación superior. Se evidencia la importancia de distribuir y gestionar los recursos financieros enfocados a la investigación científica como instrumento para enfrentar los nuevos retos y cambios en el sector de la educación.

\section{REFERENCIAS}

Álvarez-Aros, E.L. y C.A. Bernal-Torres, Modelo de Innovación Abierta: Énfasis en el Potencial Humano, doi.org/10.4067/S0718-07642017000100007. Inf. tecnol. [online], 28(1), 65-76 (2017).

Becker, G. Human Capital: A theoretical and empirical analysis, with special reference to education- National Bureau of Economic. 3를. Ed., 1-412, ISBN: 0-226-04119-0. Research. N. 80, Series Generales, Estados Unidos (1994).

Bezhani, I., Intellectual capital reporting at UK universities. doi/full/10.1108/14691931011039679. Journal of Intellectual Capital, 11(2), 2, 179-207 (2010).

Bueno, E., C. Murcia, M. Longo y C. Merino, Modelo intellectus: Medición y gestión del capital intelectual. CIC., 3-60, IADE. Madrid (2011).

Carro-Suárez, J., S. Sarmiento-Paredes y G. Rosano-Ortega, La cultura organizacional y su influencia en la sustentabilidad empresarial. La importancia de la cultura en la sustentabilidad empresarial. doi.org/10.1016/j.estger.2017.11.006. Estudios Gerenciales Universidad ICESI, 33 (145) 352-365 (2017).

Casanueva, C., y A. Gallego, Social Capital and Innovation: An Intra-departmental Perspective. DOI: 10.2307/41783644. Management Revue, 21(2), 135-154 (2010).

Colunga, A., V. Molina y E. Díaz, Modelo de Gestión Innovadora en un Centro Tecnológico Mexicano de Investigación y Desarrollo. doi.org/10.4067/S0718-07642018000300121. Inf. tecnol. 29(3), 121-132 (2018).

Cortez, D., Medir la producción científica de los investigadores universitarios: La bibliometría y sus límites. Revista de Educación Superior. XXVI (2)-142, 43-65 (2007).

Cypher, J. M. y J. Dietz, The process of economic development. 3를 Ed. ISBN: 780415771030-97804157710479780203895061. Routledge. New York (2014).

Dean, A. y M. Kretschmer, Can ideas capital? Factors of production in the Postindustrial Economy: A review and Critique. doi.org/10.5465/amr.2007.24351866. Academy of Man Agreement Review 32 (2) 573-594 (2007).

Diez, J., M. Ochoa, B. Prieto y A. Santidrian, Intellectual Capital and Value Creation in Spanish Firms. doi.org/10.1108/14691931011064581 Journal of Intellectual Capital, 11(3), 348-367 (2010).

Espejo, R. L., Interacción Simbólica en un Acto de Subjetividad de Género., doi.org/10.4067/S071850062015000500006. Formación Universitaria, 8(5) 43-58 (2015).

González, J. y T. Rodríguez, Modelos de Capital Intelectual y sus indicadores en la universidad pública. Cuadernos de administración, 26 (43), 113-128 (2010).

Gujarati, D. y D. Porter, Econometría. 5aㅗ Ed., 673-773, ISBN: 978-607-15-0294-0. McGraw Hill, Bogotá, Colombia. (2010).

Hernández, L., Y. Piñeiro y F. Ciudad, Sistema de actividades de formación para la aplicación de la gestión del conocimiento en el Centro de Innovación y Calidad de la Educación. Revista Cubana de ciencias informáticas, 9 (3) 128137 (2015).

Labra, R. y P. Sánchez, National intellectual capital assessment models: a literature review. doi.org/10.1108/JIC-112012-0100. Journal of Intellectual Capital, 14(4), 582-607 (2013). 
Lagrosen, S., R. Seyyed-Hashemi y M. Leitner, Examination of the dimensions of quality in higher education, doi.org/10.1108/09684880410536431. Quality Assurance in Education., 12(2) ,61-69 (2004).

Martín-de-Castro, G., M. Delgado-Verde, P. López-Sáez y J. Navas-López, Towards 'An Intellectual Capital-Based View of the Firm': Origins and Nature. DOI 10.1007/s10551-010-0644-5. Journal of Business Ethics 98 (4), 649-662. (2011).

Martínez, M., A procedure to design a structural and measurement model of intellectual capital: An exploratory study. doi.org/10.1016/j.im.2006.03.002.Information \& Management, 43(5) 617-626 (2014).

Marulanda, C., M. Lopez y F. López, La Cultura Organizacional y las Competencias para la Gestión del Conocimiento en las Pequeñas y Medianas Empresas (PYMEs) de Colombia. doi.org/10.4067/S0718-07642016000600002. Inf. tecnol. [Online]. 27(6) 03-10 (2016).

Mercado-Salgado, P., Validez inicial de una escala de medición del capital intelectual en universidades, doi.org/10.11144/Javeriana.upsy15-2.viem.Universitas Psychologica, 15(2), 109-119 (2016)

Min-Wei, L. y B. Barry, Researchers' Industry Experience and Productivity in University-Industry Research Centers: $A$ "Scientific and Technical Human Capital" Explanation. doi.org/10.1007/s10961-005-6111-2. The Journal of Technology Transfer, 31 (2), 269-290 (2006).

Moon, Y. y H. Kym, A model for the value of intellectual capital. DOI: 10.1111/j.1936-4490. 2006.tb00630. x. Journal of Administrative Sciences. 23 (3), 253-269 (2006).

Ríos, J. A. y M.L. Castillo, Efectos de la capacidad innovadora en el crecimiento económico. Análisis comparativo entre Países desarrollados y en desarrollo. Región y sociedad. 27 (64), 110-138 (2015).

Rojas M. y R. Espejo, Método de Ecuaciones Estructurales Econométricas para el Análisis de Gestión en Ciencia. doi.org/10.4067/S0718-07642018000500215.Información Tecnológica. 29(5), 215-226 (2018).

Sánchez, M.P., S. Elena y R. Castrill, Intellectual capital dynamics in universities: a reporting model. doi.org/10.1108/14691930910952687. Journal of Intellectual Capital, 10(2), 307-324 (2009).

Secundo, G., A. Margherita, G. Elia y G. Passiante, Intangible Assets in Higher Education and Research: Mission, Performance or Both? doi.org/10.1108/14691931011039651. Journal of Intellectual Capital. 11(2), 140-157 (2010).

Wann-Yih, W., C. Man-Ling y C. Chih-Wei, Promoting Innovation through the accumulation of Intellectual Capital, Social Capital, and Entrepreneurial Orientation. doi.org/10.1111/1467-9914.00120-i1. R\&D Management. 38(3). 265-277 (2008).

Yi-Chu, H. y J. Yen-Chun, Intellectual capital and knwoledge productivity: The Taiwan biotech industry. doi.org/10.1108/00251741011041364. Management Decision, 48 (4) 580-599 (2010)

Zlatea, S. y C. Enache, The interdependence between human capital and organizational performance in higher education. doi.org/10.1016/j.sbspro.2015.02.096. Procedia - Social and Behavioral Sciences 180 (5), 136-143 (2015). 
